\title{
Abdominal compartment syndrome - the prevention and treatment of possible lethal complications following hip arthroscopy: a case report
}

\author{
Kinga Ciemniewska-Gorzela ${ }^{1 *}$, Tomasz Piontek ${ }^{1}$ and Andrzej Szulc ${ }^{2}$
}

\begin{abstract}
Introduction: Intra-abdominal hypertension and abdominal compartment syndrome have been increasingly recognized as a hip arthroscopy complication over the past decade. In the absence of consensus definitions and treatment guidelines, the diagnosis and management of intra-abdominal hypertension and abdominal compartment syndrome remains variable from institution to institution.

Case presentation: We report the occurrence of the extravasation of fluid into the abdomen during arthroscopic treatment of femoroacetabular impingement combined with resection of trochanteric bursa and our management of the condition in a 55-year old Caucasian woman.

Conclusions: We present an algorithm of treatment of abdominal compartment syndrome, as a hip arthroscopy complication, according to the consensus definitions and recommendations of the World Society of the Abdominal Compartment Syndrome. In the algorithm options, we have included paracentesis and percutaneous catheter decompression as the main point of treatment. Our algorithm will have a broader clinical impact on orthopedic surgery, anesthesiology and emergency medicine.
\end{abstract}

Keyword: Abdominal compartment syndrome, Complication, Hip arthroscopy

\section{Introduction}

By having an upgraded understanding of hip anatomy, arthroscopy has been established as a standard technique in hip surgery. The potential complications of hip arthroscopy are: neurovascular traction injury, direct trauma to neurovascular structures, compression injury to the perineum, traction fixation device injury, arthroscopy-related trauma, breakage of instruments, vascular insult to the femoral head, infection, and fluid extravasations $[1,2]$. The development of the hemodynamically compromising abdominal compartment syndrome (ACS) after hip arthroscopy is a rare but possible and lethal complication [1-8]. Early signs of abdominal pain due to the accumulation of fluid in the abdominal cavity may be masked by regional

\footnotetext{
* Correspondence: kinga.ciemniewska@rehasport.pl

'Rehasport Clinic Poznan, Clinic of Pediatric Orthopedic Surgery UM in Poznan, Rehasport Clinic, ul. Górecka 30, 60-201 Poznań, Poland Full list of author information is available at the end of the article
}

anesthesia and sedation [5]. Pathophysiologically, it deranges cardiovascular hemodynamics, respiratory and renal functions, and may eventually lead to multi-organ failure [9-12]. Significant progress has been made over the past decade towards understanding the etymology and pathophysiology surrounding intra-abdominal hypertension (IAH) and ACS. The absence of consensus definitions and treatment guidelines, however, has led to confusion over both the prevalence of IAH and ACS, as well as the most effective treatment strategies for such patients. Failure to recognize and appropriately treat ACS is uniformly fatal, whereas, prevention and/or timely intervention has been associated with marked improvements in organ function, and overall patient survival [9-11]. The survey experts in the field of hip arthroscopy from the Multicenter Arthroscopy of the Hip Outcomes Research Network (MAHORN) Group had determined the frequency of symptomatic intra-abdominal fluid extravasation (IAFE) 
after arthroscopic hip procedures. Potential risk factors had been identified among 40 cases of symptomatic IAFE [13]. The main disadvantage of the study had been the fact that the recommended strategies of treatment were based on the experience of experts in the field of hip arthroscopy and not in the field of the World Society of the Abdominal Compartment Syndrome (WSACS). Moreover, the study had not presented a clear algorithm of treatment, which is very important to avoid mistakes in emergency treatment.

The presented case highlights the need for surgeons to be aware of this rare but serious complication, and we indicate the steps necessary to manage it promptly.

In this manuscript we aim to report a life-threatening case of primary acute ACS resulting from iatrogenic extravasation after hip arthroscopy. We also reviewed the literature and created an algorithm of treatment of ACS according to the WSACS, and additionally reviewed consensus definitions and recommendations.

\section{Case presentation}

A 55 -year-old Caucasian woman $(53 \mathrm{~kg}, 160 \mathrm{~cm}$; body mass index $20.7 \mathrm{~kg} / \mathrm{m}^{2}$ ) had been scheduled for right hip arthroscopy, partial labral resection for a labral tear, psoas release of her right hip, and resection of trochanteric bursa in relation to the gluteus medius tendon. She consented to undergo spinal anesthesia and pharmacological sedation with propofol in constant 10 to $15 \mathrm{~mL} /$ hour infusion for this procedure. Standard monitoring (electrocardiogram), noninvasive blood pressure monitoring and pulse oximetry were used. Following uneventful spinal anesthesia, at the fourth lumbar interspace using $3 \mathrm{~mL}$ isobaric bupivacaine $0.5 \%$, she was positioned supine, prepped, and draped. Supplemental oxygen had been provided by a nasal cannula. An infusion pump with propofol had been admitted. Traction had been applied to the lower extremities and was confirmed by a fluoroscopic examination. The anterior and anterolateral arthroscopic portals were used as standard approaches to the right hip. The arthroscope and arthroscopic instruments were introduced into the joint under fluoroscopic guidance, and $0.9 \%$ sodium chloride. Interportal capsulotomy had been performed to facilitate instrument navigation and arthroscopic visualization. After hip diagnostic arthroscopy the damaged labrum had been debrided back to stable margins. The acetabular rim had been adjusted with an arthroscopic bar. The psoas tendon had been released at the level of the capsule via a transcapsular approach. The femoroplasty for the decompression of the small cam deformity had been performed.

The DYONICS 25 Fluid Management System was used. This high-flow pump pumps fluid with a maximum flow rate of $1.5 \mathrm{~L} /$ minute for procedural speed and efficiency. A total of eight $3 \mathrm{~L}$ bags were used for the procedure. The procedure lasted approximately 2 hours, and anesthetic management was uneventful until the final 5 minutes of surgery. To ensure no increases in abdominal tension, we had performed a preoperative abdominal routine examination under anesthesia before prepping and draping, in order to obtain a baseline followed by a series of abdominal examinations throughout the procedure. The remaining 10 minutes of surgery had been devoted to work outside of the joint in paratrochanteric bursa and gluteus medius origin. At the final stage of surgery she became acutely short of breath with a tachycardia above 100. Acute abdominal pain was visible. The upper body warming blanket had been removed, revealing significant abdominal distension. Systolic blood pressure was low at approximately $90 \mathrm{mmHg}$. The surgeon was notified and surgical closure procedures swiftly completed the operation. The incisions were closed, and preparations were made to transfer her to the recovery room. She remained hemodynamically stable for the rest of the procedure. Although the cause of abdominal distension was not known at that point, there was an assumption that it had resulted from either large amounts of blood or arthroscopy irrigation fluid, or both, in her abdomen. A general surgeon was consulted; the surgeon recommended ultrasonographic assessment of her abdomen for an evaluation of the type and quantity of fluid. The ultrasonographic examination was performed and clear fluid was found. Right lower quadrant paracentesis was performed and 3L of the clear, colorless fluid was drained from her abdomen, showing an immediate improvement in her hemodynamic status.

\section{The technique of paracentesis}

She was positioned in a semi-sitting position tilt, on her right side. Her abdomen was sterile. Right lower quadrant palpation had been performed before the peritoneal fluid removal. The sterile technique of the paracentesis was done using a 14G catheter. The catheter had been carefully percutaneously inserted into the abdominal cavity and left in situ for slow drainage.

\section{Discussion}

According to WSACS definitions, primary ACS (formerly termed as surgical, postoperative, or abdominal ACS) is characterized by the presence of acute, or subacute IAH, of relatively brief duration $[9,10]$. Such duration occurs as a result of an intra-abdominal reason such as: abdominal trauma, ruptured abdominal aortic aneurysm, hemoperitoneum, acute pancreatitis, secondary peritonitis, retroperitoneal hemorrhage, or liver transplantation. Fluid extravasations after hip arthroscopy also can lead to ACS $[9,10]$. This potentially lethal complication of hip arthroscopy highlights the need for orthopedists to be aware of this rare but serious complication, and of the steps needed to be undertaken in order to manage it promptly. Although Fowler and Owens [6] mention death 
after hip arthroscopy among Bartlett's patients, Bartlett et al. [3] do not confirm such information in their original paper. The prevalence and etiological factors for IAH and ACS after hip arthroscopy are being reviewed.

Evidence-based medicine treatment guidelines have been presented to facilitate the diagnosis and management of IAH and ACS (Figure 1). The choice (and success) of the medical management strategies given in Figure 1 is strongly related to both the etymology of the patient's IAH/ACS, and the patient's clinical situation. The appropriateness of each intervention should always be considered prior to implementing these interventions in any individual patient. This paper presents the case of ACS after hip arthroscopy, treated successfully with paracentesis and percutaneous catheter decompression. The pathophysiology of IAH and ACS in hip arthroscopy has been described in many papers [3-8]. The details have been collected in Tables 1 and 2. Many authors have suggested the only possible way for the irrigation fluid to flow is by a retroperitoneal course along the iliopsoas muscle and the iliac vessels with intraperitoneal perforation along their course [4-8]. The proposed mechanism

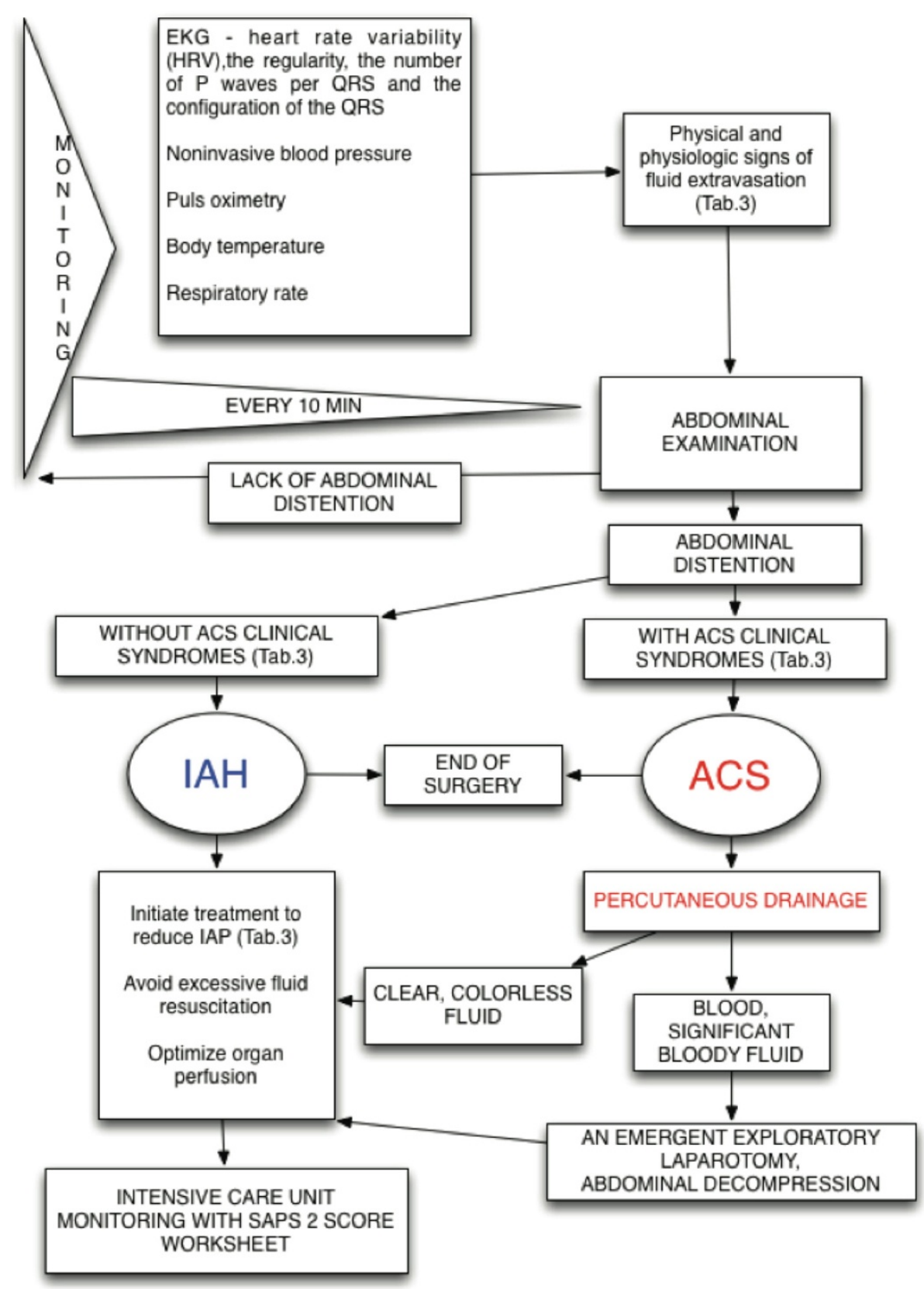

Figure 1 Intra-abdominal hypertension/intra-abdominal compartment syndrome management algorithm. 
Table 1 Cases of fluid extravasation after hip arthroscopy - demographic data and hip operative procedure

\begin{tabular}{|c|c|c|c|}
\hline $\begin{array}{l}\text { Authors, year of publication } \\
\text { and reference number }\end{array}$ & Age and gender & Hip treatment procedures & $\begin{array}{l}\text { Time of surgery (minutes) } \\
\text { and surgical position }\end{array}$ \\
\hline Bartlett et al., 1998 [3] & 50-year-old man & $\begin{array}{l}\text { 1. Loose-body removal } 13 \text { days after acetabular } \\
\text { fracture of both columns treated with open } \\
\text { reduction-internal fixation using an } \\
\text { ilioinguinal approach }\end{array}$ & 135/lateral position \\
\hline \multirow[t]{2}{*}{ Haupt et al., 2008 [4] } & 15-year-old girl & 1. Capsulotomy & 105/lateral position \\
\hline & & $\begin{array}{l}\text { 2. Adhesion releases after open acetabular } \\
\text { retroversion corrected by trimming the } \\
\text { anterosuperior rim with reattachment } \\
\text { of the labrum. }\end{array}$ & \\
\hline \multirow[t]{3}{*}{ Sharma et al., 2009 [5] } & 45-year-old woman & 1. Limited capsulectomy & 160/supine position \\
\hline & & 2. Labral repair & \\
\hline & & 3. Psoas release & \\
\hline \multirow[t]{3}{*}{ Fowler and Owens, 2010 [6] } & 42-year-old man & 1. Limited capsulectomy & 95/lateral position \\
\hline & & 2. Psoas tenotomy & \\
\hline & & $\begin{array}{l}\text { 3. Debridement of the anterior and superior } \\
\text { labrum and pincer-type lesion }\end{array}$ & \\
\hline \multirow[t]{3}{*}{ Verma and Sekiya, 2010 [7] } & 21-year-old woman & 1. Capsulotomy & 139/supine position \\
\hline & & 2. Iliopsoas tenotomy & \\
\hline & & $\begin{array}{l}\text { 3. Osteoplasty to treat the femoroacetabular } \\
\text { cam impingement }\end{array}$ & \\
\hline \multirow[t]{4}{*}{ Ladner et al., 2010 [8] } & 42-year-old woman & 1. Limited capsulectomy & 165/supine position \\
\hline & & 2. Debridement of the irreparable large labral tear & \\
\hline & & 3. Chondroplasty on the acetabular rim & \\
\hline & & $\begin{array}{l}\text { 4. Osteoplasty of the femoral head-neck junction } \\
\text { and acetabular rim }\end{array}$ & \\
\hline \multirow[t]{4}{*}{ Current case } & 55-year-old woman & 1. Capsulotomy & 120/supine position \\
\hline & & 2. Iliopsoas tenotomy & \\
\hline & & $\begin{array}{l}\text { 3. Osteoplasty to treat the femoroacetabular } \\
\text { pincer and cam impingement }\end{array}$ & \\
\hline & & $\begin{array}{l}\text { 4. Resection of trochanteric bursa in relation } \\
\text { to gluteus medius tendon }\end{array}$ & \\
\hline
\end{tabular}

of extravasation is similar to our case. After having performed an analysis of all the cases described in the literature, and having collected data from our case, we were unable to indicate any clear risk factors of IAH and ACS in hip arthroscopy. In contrast to other authors' suggestions, a prolonged surgical time ( $95-165 \mathrm{~min}$ in analyzed cases), and patient's position (four cases in supine positions and three cases in lateral positions) or specific surgical procedures such as extra-articular surgery (many types of surgical procedures), we could define no clear risk factors of IAH or ACS. We agree with Verma and Sekiya [7], and hypothesize that our patient had had communication between the retroperitoneal and peritoneal cavity, which allowed for this amount of fluid extravasation. This hypothesis is also supported by nephrologists' experience with acute ultrafiltration failure (AUFF), which is an important clinical problem among patients having peritoneal dialysis (PD). Lam et al. reported the cases of three patients on continuous ambulatory PD who developed reversible ultrafiltration failure secondary to retroperitoneal leakage (RPL) [14]. Lam et al. also reported that during the 5-year study period, 36 patients in a cohort of 743 patients on maintenance PD developed AUFF. Of these 36 patients, 23 were found to have RPL, which was confirmed by either computed tomography $(n=16)$ or magnetic resonance imaging $(n=7)$ [15]. The authors concluded that RPL was not uncommon among patients with AUFF. There is a high possibility for the occurrence of RPL among patients with AUFF and additionally having a history of hernia or pleuroperitoneal communication. In addition, the commonly used high-flow pump for hip arthroscopy can pump fluid with a maximum flow rate of $1.5 \mathrm{~L} /$ minute; the catheter for PD can transmit fluid with a maximum flow of $0.5 \mathrm{~L} /$ minute and would fill up the abdomen with $2 \mathrm{~L}$ of fluid in 5 to 10 minutes. This means that even 2 to 3 minutes of 
Table 2 Cases of fluid extravasation after hip arthroscopy - intra-abdominal hypertension/abdominal compartment syndrome treatment and outcomes

Author and Reference Intra-abdominal hypertension/acute abdominal compartment syndrome clinical Intra-abdominal hypertension/acute Treatment results

number signs

abdominal compartment syndrome

Abdomen

treatment procedures

Significant abdominal distension

Others

1. Nonoperative medical management

Despite prolonged asystole, the patient

2. An emergent exploratory laparotomy closed primarily

Haupt et al. [4] Diffuse abdominal pain 4 hours after 1. Body temperature decreased from $36.3^{\circ}$ to surgery $34.5^{\circ} \mathrm{C}$ at the end of the operation

Nonoperative medical management

2. Concurrent neurologic symptoms, resembling absence seizures occurred

1. Acute hypotensive with a systolic blood pressure of $60-70 \mathrm{mmHg}$

2. Unresponsive

3. Apnoeic

4. Lower extremities appeared cyanotic no pulse could be palpated in either leg

Verma and Sekiya [7] Distended and firm abdomen but easily compressible

1. Elevated bladder pressures $(42 \mathrm{mmHg})$

2. An increased peak inspiratory pressure, thus preventing extubation

1. Nonoperative medical management

2. Urgent mini-laparotomy and then diagnostic laparotomy

1. Nonoperative medical management

2. An emergent exploratory laparotomy. The abdomen was left open, and a wound vacuum was placed.

Nonoperative medical management

1. Hypothermia during the surgical procedure

2. Right labia was asymmetrically enlarged

Ladner et al. [8] Abdomen noticeably distended

1. Core body temperature remained above $36.8^{\circ} \mathrm{C}$

2. At no time was her respiratory or cardiac function compromised based on clinical examination, blood pressure, heart rate, arterial blood gas values, and electrocardiographic data

1. Acute hypotension with a systolic blood pressure of $60-70 \mathrm{mmHg}$

Paracentesis - no fluid was obtained. A computed tomography scan after paracentesis showed a copious amoun of fluid in the intraperitoneal area and small amount in the retroperitoneal

2. Unresponsiveness

Paracentesis and percutaneous slow drainage

Immediate improvement in the patient's hemodynamic status

Asymptomatic in his right hip and groin but is continuing follow-up by a general surgeon for abdominal complaints related syndrome

The irrigation solution was absorbed

The irrigation solution was absorbed the next day 
surgery without abdomen examinations may be sufficient to overlook dangerous abdominal extravasation.

In our opinion, hip arthroscopy per se, places the patient at high risk for primary ACS. Intra-abdominal fluid extravasation is a known complication ( $0.16 \%$ incidence) of hip arthroscopy which needs to be informed to the patient prior to the procedure.

The indication of risk factors of IAH and ACS in hip arthroscopy needs further research on a larger scale of cases. It appears that patients with a history of hernia or pleuroperitoneal communication are more likely to be classified as at higher risk of ACS before hip arthroscopy. However, complications are very rare, and because of that we recommend to collect all cases in a WSACS database, or precisely describe the cases in medical papers. This should allow us to collect a larger amount of data for further analysis. The primary mission of WSACS is to promote research, foster education, and improve the survival rate of patients with IAH and ACS, by sharing information on effective management strategies [9,10]. The WSACS provides instructional materials (IAH and ACS consensus definitions and clinical practice guidelines) to upgrade the education of physicians, nurses, respiratory therapists, and other healthcare providers [16]. WSACS has recently published two consensus documents detailing the current state-of-the-art diagnosis and management of IAH/ACS $[9,10]$. No single management strategy can be uniformly applied to a patient with IAH/ACS. Several fundamental management concepts, however, remain appropriate across all cases. While surgical decompression is commonly considered the only treatment for IAH/ACS, nonoperative medical management strategies are now recognized as playing a vital role in both the prevention and treatment of organ dysfunction and failure due to elevated intra-abdominal pressure (IAP) $[11,12]$. Appropriate treatment and management of IAH and/or ACS is based upon four general principles: (a) serial monitoring, (b) optimization of systemic perfusion and a patient's organ function with elevated IAP, (c) institution of specific medical procedures (with paracentesis and percutaneous catheter decompression as a main point of treatment) to reduce IAP and the end-organ consequences of IAH/ACS, and (d) prompt surgical decompression for refractory IAH $[9,10]$. An algorithm for the management of a patient with IAH/ACS has been proposed in Table 3.

Among patients having undergone trauma or elective surgery, operative decompression is advocated if noninvasive treatment options prove insufficient. Decompression laparotomy reduces IAP instantaneously and is often a life-saving procedure [9-12]. Given the morbidity of open abdominal decompression, less invasive means to reduce IAP would certainly be appealing. Percutaneous catheter decompression represents a less invasive method for treating IAH or primary ACS due to free intra-abdominal fluid. This technique appears to be effective in reducing IAP, and potentially corrects the IAH-induced organ dysfunction. Possible performance under ultrasound, or computed tomography guidance and percutaneous decompression among appropriate patients, appears to be effective in solving IAH/ACS and avoiding the need for surgical decompression [11,12]. In light of the potential benefits of avoiding abdominal

Table 3 Algorithm-related physical and physiological signs of fluid extravasation syndrome and abdominal compartment syndrome: initiated treatment options to reduce intra-abdominal pressure

Physical and physiologic Abdominal compartment syndrome clinical Initiate treatment to reduce intra-abdominal pressure signs of fluid extravasation syndromes \begin{tabular}{ll}
\hline Cardiac arrhythmias & Systolic blood pressure less than $90 \mathrm{mmHg}$ or \\
need for catecholamine support
\end{tabular}

Hypotension

Oxygen saturation $<95$

Need for hemodialysis or creatinine level greater than $177 \mathrm{umol} / \mathrm{L}$ after rehydration

Hypothermia $($ core
temperature $\left.<35^{\circ} \mathrm{C}\right)$

Unresponsiveness

Shortness of breath

Shortness of breath/apnea
Improve abdominal wall compliance

Sedation and analgesia. Neuromuscular blockade. Avoid head of bed $>30$ degrees

$\mathrm{PaO}_{2} 60 \mathrm{mmHg}$ or less or need for mechanical Correct positive fluid balance

Avoid excessive fluid resuscitation. Diuretics. Colloids/hypertonic fluids. Hemodialysis/ultrafiltration

Organ support. Maintain APP $>60 \mathrm{mmHg}$ with vasopressors. Optimize ventilation, alveolar recruitment. Use transmural airway pressures

Pplattm = Pplat - IAP. Consider using volumetric preload indices. If using PAOP/CVP, use transmural pressures

PAOPtm $=$ PAOP $-0.5 \times I A P C V P t m=C V P-0.5 \times I A P$

Evacuate intraluminal contents. Nasogastric decompression. Rectal decompression. Gastro-/colo-prokinetic agents

Peak pressure $\uparrow$ on artificial ventilation

Abbreviations: APP abdominal perfusion pressure, $C V P$ central venous pressure, IAP intra-abdominal pressure, $P a O_{2}$ oxygen partial pressure arterial, $P A O P$ pulmonary artery occlusion pressure, Pplat plateau pressure, Pplattm transmural plateau pressure, $t m$ transmural. 
decompression, we suggest that percutaneous catheter decompression should be considered among patients with intraperitoneal fluid or blood, who demonstrate symptomatic IAH or ACS.

\section{Conclusions}

We have presented an algorithm of treatment of ACS, as a hip arthroscopy complication, according to the WSACS consensus definitions and recommendations. In the algorithm options we have included paracentesis and percutaneous catheter decompression as a main point of treatment. The authors anticipate that these definitions and recommendations will be dynamic and will change as new research is published. They will have a broader clinical impact across orthopedic surgery, anesthesiology and emergency medicine.

\section{Consent}

Written informed consent has been obtained from the patient for publication of this case report and any accompanying images. A copy of the written consent is available for review by the Editor-in-Chief of this journal.

\section{Abbreviations}

ACS: Abdominal compartment syndrome; AUFF: Acute ultrafiltration failure; IAFE: Intra-abdominal fluid extravasation; IAH: Intra-abdominal hypertension; IAP: Intra-abdominal pressure; MAHORN: Multicenter Arthroscopy of the Hip Outcomes Research Network Group; PD: Peritoneal dialysis;

RPL: Retroperitoneal leakage; WSACS: World Society of the Abdominal Compartment Syndrome.

\section{Competing interests}

The authors declare that they have no competing interests.

\section{Authors' contributions}

KC-G participated in the study design, sequence alignment, data interpretation and literature search, and drafted the manuscript. TP participated in study design, sequence alignment, data collection and interpretation, and coordination to draft the manuscript. ASz participated in the study design, data interpretation and helped to draft the manuscript. All authors have read and approved the final manuscript.

\section{Acknowledgment}

Many thanks to our patient who consented to the publication of this case report.

\section{Author details}

'Rehasport Clinic Poznan, Clinic of Pediatric Orthopedic Surgery UM in Poznan, Rehasport Clinic, ul. Górecka 30, 60-201 Poznań, Poland. ${ }^{2}$ Clinic of Pediatric Orthopedic Surgery UM in Poznan, Clinic of Pediatric Orthopedic Surgery, ul 28 czerwca 1956 r. 134/145, 61-495 Poznań, Poland.

Received: 17 April 2014 Accepted: 16 September 2014 Published: 14 November 2014

\section{References}

1. Funke EL, Munzinger U: Complications in hip arthroscopy. Arthroscopy 1996, 12:156-159.

2. Sampson TG: Complications of hip arthroscopy. Tech Orthop 2005, 20:63-66

3. Bartlett CS, DiFelice GS, Buly RL, Quinn TJ, Green DS, Helfet DL: Cardiac arrest as a result of intraabdominal extravasation of fluid during arthroscopic removal of a loose body from the hip joint of a patient with an acetabular fracture. J Orthop Trauma 1998, 12:294-299.
4. Haupt U, Volkle D, Waldherr C, Beck M: Intra- and retroperitoneal irrigation liquid after arthroscopy of the hip joint. Arthroscopy 2008, 24:966-968.

5. Sharma A, Sachdev H, Gomillion M: Abdominal compartment syndrome during hip arthroscopy. Anaesthesia 2009, 64:567-569.

6. Fowler J, Owens BD: Abdominal compartment syndrome after hip arthroscopy. Arthroscopy 2010, 26:128-130.

7. Verma M, Sekiya JK: Intrathoracic fluid extravasation after hip arthroscopy. Arthroscopy 2010, 26:90-94.

8. Ladner B, Nester K, Cascio B: Abdominal fluid extravasation during hip arthroscopy. Arthroscopy 2010, 26:131-135.

9. Malbrain ML, Cheatham ML, Kirkpatrick A, Sugrue M, Parr M, De Waele J, Balogh Z, Leppäniemi A, Olvera C, Ivatury R, D'Amours S, Wendon J, Hillman K, Johansson K, Kolkman K, Wilmer A: Results from the International Conference of Experts on Intra-abdominal Hypertension and Abdominal Compartment Syndrome. I. Definitions. Intensive Care Med 2006, 32:1722-1732.

10. Cheatham ML, Malbrain ML, Kirkpatrick A, Sugrue M, Parr M, De Waele J, Balogh Z, Leppäniemi A, Olvera C, Ivatury R, D'Amours S, Wendon J, Hillman K, Wilmer A: Results from the International Conference of Experts on Intra-abdominal Hypertension and Abdominal Compartment Syndrome. II. Recommendations. Intensive Care Med 2007, 33:951-962.

11. Deslauriers NR, Denault A: Acute abdominal compartment syndrome. Can J Anesth/J Can Anesth 2009, 56:678-682.

12. Souadka A, Mohsine R, Ifrine L, Belkouchi A, El Malki HO: Acute abdominal compartment syndrome complicating a colonoscopic perforation: a case report. J Med Case Reports 2012, 6:51.

13. Kocher MS, Frank JS, Nasreddine AY, Safran MR, Philippon MJ, Sekiya JK, Kelly BT, Byrd JW, Guanche CA, Martin HD, Clohisy JC, Mohtadi NG, Griffin DR, Sampson TG, Leunig M, Larson CM, llizaliturri VM Jr, McCarthy JC, Gambacorta PG: Intra-abdominal fluid extravasation during hip arthroscopy: a survey of the MAHORN group. Arthroscopy 2012, 28:1654-1660.

14. Lam MF, Lo WK, Chu FS, Li FK, Yip TP, Tse KC, Chan TM, Lai KN: Retroperitoneal leakage as a cause of ultrafiltration failure. Perit Dial Int 2004, 24:466-470.

15. Lam MF, Lo WK, Tse KC, Yip TP, Lui SL, Chan TM, Lai KN: Retroperitoneal leakage as a cause of acute ultrafiltration failure: its associated risk factors on peritoneal dialysis. Perit Dial Int 2009, 29:542-547.

16. WSACS - The Abdominal Compartment Society: IAH and ACS consensus definitions and clinical practice guidelines. [http://www.wsacs.org/]

doi:10.1186/1752-1947-8-368

Cite this article as: Ciemniewska-Gorzela et al:: Abdominal compartment syndrome - the prevention and treatment of possible lethal complications following hip arthroscopy: a case report. Journal of Medical Case Reports 2014 8:368.

\section{Submit your next manuscript to BioMed Central and take full advantage of:}

- Convenient online submission

- Thorough peer review

- No space constraints or color figure charges

- Immediate publication on acceptance

- Inclusion in PubMed, CAS, Scopus and Google Scholar

- Research which is freely available for redistribution 